Revue internationale P.M.E.

Économie et gestion de la petite et moyenne entreprise

\title{
PME et entrepreneuriat dans la littérature francophone stratégique
}

\section{Jean-Pierre Boissin, Jean-Claude Castagnos et Gilles Guieu}

Volume 13, numéro 1, 2000

URI : https://id.erudit.org/iderudit/1008669ar

DOI : https://doi.org/10.7202/1008669ar

Aller au sommaire du numéro

Éditeur(s)

Presses de l’Université du Québec

ISSN

0776-5436 (imprimé)

1918-9699 (numérique)

Découvrir la revue

Citer cet article

Boissin, J.-P., Castagnos, J.-C. \& Guieu, G. (2000). PME et entrepreneuriat dans la littérature francophone stratégique. Revue internationale P.M.E., 13(1), 39-64. https://doi.org/10.7202/1008669ar
Résumé de l'article

Cet article analyse la production francophone en stratégie publiée entre 1990 et 1995 sur les thèmes de la PME et de l'entrepreneuriat dans sept supports édito riaux: Revue internationale PME, Revue d'économie industrielle, Revue française de gestion, Économies et sociétés, Gestion 2000, Gestion et les deux premiers tomes de Perspectives en management stratégique. Les 60 articles analysés présentent des traits spécifiques : une production concentrée sur des auteurs et des centres dominants; des recherches axées sur trois thèmes privilégiés (processus, technologie, dirigeants) ; des méthodes souvent explicitées attestant d'un éclectisme méthodologique. Les réseaux de cocitations fondant les bibliographies des articles renseignent sur la généalogie des thématiques de la PME et de rentrepreneuriat et permettent d'identifier révolution récente de la recherche sur les PME.
Ce document est protégé par la loi sur le droit d'auteur. L'utilisation des services d’Érudit (y compris la reproduction) est assujettie à sa politique d'utilisation que vous pouvez consulter en ligne.

https://apropos.erudit.org/fr/usagers/politique-dutilisation/ 


\title{
PME et entrepreneuriat dans la littérature francophone stratégique
}

\author{
Jean-Pierre BOISSIN \\ Jean-Claude CASTAGNOS \\ Gilles GUIEU \\ CERAG, Université Pierre-Mendès-France
}

\section{MOTS CLÉS}

\section{Stratégie - PME - Entrepreneuriat - Analyse scientométrique Bibliographie - Structuration sociocognitive}

\begin{abstract}
RÉSUMÉ
Cet article analyse la production francophone en stratégie publiée entre 1990 et 1995 sur les thèmes de la PME et de l'entrepreneuriat dans sept supports éditoriaux: Revue internationale PME, Revue d'économie industrielle, Revue française de gestion, Économies et sociétés, Gestion 2000, Gestion et les deux premiers tomes de Perspectives en management stratégique. Les 60 articles analysés présentent des traits spécifiques: une production concentrée sur des auteurs et des centres dominants; des recherches axées sur trois thèmes privilégiés (processus, technologie, dirigeants); des méthodes souvent explicitées attestant d'un éclectisme
\end{abstract}

\section{LES AUTEURS}

Jean-Pierre Boissin est maître de conférences à l'UFR DGES de l'Université Pierre-MendèsFrance, et s'intéresse aux processus de croissance des firmes et au gouvernement d'entreprise. Téléphone: 04768254 14. Télécopieur : 04768259 99. Courriel : boissin @esa.upmf-grenoble.fr

Jean-Claude Castagnos, chargé de recherche au CNRS, est détaché auprès de l'École supérieure des affaires de Grenoble. Spécialiste en gestion de la formation et de la recherche, il a ultérieurement traité de nombreux sujets dont l'innovation, la relation structure-efficacité, la gestion des rémunérations. Téléphone : 04768256 15. Télécopieur : 04768259 99. Courriel: castagno@esa.upmf-grenoble.fr

Gilles Guieu est maître de conférences à l'Institut universitaire de technologie (IUT) de Valence, composante de l'Université Pierre-Mendès-France. Ses articles portent notamment sur les processus de prise de contrôle. Téléphone : 0492614866 . Télécopieur: 0492614866. Courriel : guieu@club-internet.fr

Adresse: B.P. 47 X, 38040 Grenoble Cedex 9, France. 
méthodologique. Les réseaux de cocitations fondant les bibliographies des articles renseignent sur la généalogie des thématiques de la PME et de l'entrepreneuriat et permettent d'identifier l'évolution récente de la recherche sur les PME.

\section{ABSTRACT}

This article analyzes the production in strategic management, issued between 1990 and 1995 on the topics of SB and entrepreneurship in six French-speaking journals (Revue internationale PME, Revue d'économie industrielle, Revue française de gestion, Économies et sociétés, Gestion 2000, Gestion) and the two first volumes of Perspectives en management stratégique. The analysis of the 60 articles shows that the production is concentrated on major authors and research centers, that research is mainly devoted to three themes (processes, technology, managers), that methods are often explicitated and cover a large spectrum of methodology practices. Cocitation clusters in bibliographies give information on the genealogy of topics in SB and entrepreneurship research. They make possible the identification of the current evolution of research in SB.

\section{RESUMEN}

Este artículo analiza la producción francofona en estrategía publicada entre 1990 y 1995, sobre las temas de las PyMEs y del aparato empresarial en siete editoriales: Revue internationale PME, Revue d'économie industrielle, Revue française de gestion, Économies et sociétés, Gestion 2000, Gestion y los dos primeros tomos de Perspectives en management stratégique. Los 60 artículos analizados presentan características específicas : una producción concentrada sobre autores y centros dominantes: investigaciones orientadas sobre temas privilegiados (procesos, tecnología, dirigentes); métodos frecuentemente explicitos que muestran un eclecticismo de las metodologias. Los redes de co-citaciones que fundan las bibliografias de los articulos informan sobre la genealogía de las tematicas de las PyMEs y del aparato empresarial y permiten identificar una reciente evolución de la investigación sobre las PyMEs.

\section{ZUSAMMENFASSUNG}

Dieser Artikel analysiert die Anzahl an Themen über KMU und Unternehmertum im frankophonen Bereich in sieben Fachmagazinen: der Revue Internationale PME, Revue d'économie Industrielle, Revue française de gestion, Économies et Sociétés, Gestion 2000, Gestion und die zwei ersten Bände Perspectives en management stratégique. Die 60 untersuchten Artikel zeigen spezielle Gesichtspunkte auf: die verfassten Artikel konzentrieren sich mehr auf den Verfasser und dominierende Zentren; die Forschung privilegiert drei Themen (Prozesse, Technologie und Führung); die vielfach erwähnten Methoden unterliegen einer gezielten Auswahl. Das Netz an Co-Zitierungen aufbauend auf der Bibliographie der Artikel gibt Auskunft über die Herkunft der Themen von KMU und des Unternehmertums. Sie erlauben die Identifikation der kürzlichen Entwicklung über die Forschung der KMU. 


\section{Introduction : la «stratégie de la PME », un champ évolutif ${ }^{1}$}

Depuis quelques années, les sociologues des sciences passent au crible la littérature scientifique de gestion; un aspect de cette investigation procède de la bibliométrie ${ }^{2}$. La mesure de l'activité scientifique (notamment les articles publiés) constitue un matériau permettant de décrire les positions relatives occupées par les auteurs, les laboratoires, les courants de recherches, dans un champ opératoire déterminé (Bourdieu, 1976).

En ce qui concerne l'entrepreneurship, divers travaux récents montrent l'intérêt de caractériser l'activité de production scientifique, tant dans le but de mieux baliser le domaine de recherche que d'identifier les clivages internes. Déry et Toulouse (1996) ont décrit sa structuration sociale, à travers l'analyse du Journal of Business Venturing. À partir des 30 travaux les plus cités entre 1986 et 1995, Béchard (1996) a repéré trois strates de connaissances en entrepreneurship: les niveaux praxéologique, disciplinaire et épistémologique. Préférant une perspective historique, Filion (1997) expose la diversité des paradigmes structurants et des définitions de l'entrepreneuriat. Si l'entrepreneurship affiche clairement une épistémologie autonome, c'est récemment que la place de la PME dans la littérature scientifique a donné lieu à des études. Par exemple, Cossette (1997) a analysé les travaux publiés par la Revue internationale PME depuis sa création et a souligné l'éclectisme méthodologique des articles (par comparaison aux articles anglophones). Depuis la création de l'Association internationale de recherche sur la PME (1995) et la tenue régulière de congrès francophones, un débat sur la question de savoir quelle problématique gouverne la recherche sur la PME a pris corps : constitue-t-elle un objet spécifique d'investigation? Alors que «le paradigme de la spécificité est l'énoncé universel qui constitue le noyau dur du programme de recherche concernant la PME » (Torrès, 1998, p. 11), le repérage des nouvelles pratiques amène l'auteur à parler de PME managériale. Les années récentes constituent donc une période de gestation durant laquelle les traits distinctifs de la PME ont été débattus. À l'heure de cette controverse, il paraît judicieux d'analyser, pour ce qui concerne le champ de la production d'articles de stratégie sur la PME ou l'entrepreneuriat, comment s'articulent paradigmes dominants et courants complémentaires. Cette analyse n'a pas pour but de définir les objets de la PME et de l'entrepreneuriat à travers des propositions théoriques fondées sur la cohérence d'une logique formelle; au contraire, son but est de synthétiser la vision proposée par les chercheurs. En effet, en présentant les principaux auteurs, les lieux de recherche, les pratiques de terrain, les références bibliographiques les plus

1. Les auteurs remercient les trois lecteurs pour leurs critiques et suggestions.

2. La bibliométrie désigne l'application des mathématiques et des méthodes statistiques à l'étude des livres, articles et autres supports de communication. 
courantes, on obtient une image du champ telle que les acteurs le dessinent. Ainsi, le contenu de cet article se distingue à la fois par son approche (restitution des positions relatives d'acteurs au sein d'un domaine de recherche) et par son étendue (les articles francophones portant sur la PME ou l'entrepreneuriat publiés en stratégie).

Nos travaux antérieurs (1997-1999) fondés sur la constitution d'une base de données ont bien cerné l'importance, dans la littérature de stratégie, de la PME et de l'entrepreneuriat : un article sur quatre parus entre 1990 et 1995 en traite (tableau 4). L'opportunité est donnée, à l'aide des outils bibliométriques, de recenser et d'analyser les pratiques et les fondements scientifiques de sorte à souligner les convergences et clivages en termes d'objets de recherche ou de paradigmes. Ainsi, quelle est la place des thèmes de l'entrepreneuriat et de la PME dans les écrits francophones de stratégie ? Plus précisément : quelle est la nature de la production scientifique de cette période (auteurs, centres de recherche, revues) ? Quelle est la teneur des articles publiés (thèmes traités, méthodes) ? Quelles sont les caractéristiques des sources bibliographiques (revues et auteurs cités, réseaux de cocitations $^{3}$ ) ? Cette triple perspective éclaire l'état actuel de la structuration ${ }^{4}$ tant sociale que cognitive de la recherche en stratégie sur l'entrepreneuriat et la PME dans la communauté francophone.

\section{La maturité de la production scientifique}

Entre 1990 et 1995, 60 articles de stratégie consacrés à la PME ou à l'entrepreneuriat ont été publiés dans les principales ${ }^{15}$ revues scientifiques francophones (RFG, REI, Économies et sociétés, Gestion 2000, Gestion, RIPME) et dans les deux premiers tomes de Perspectives en management stratégique (voir liste à l'annexe I). Ces thèmes apparaissent dans près de $25 \%$ des articles francophones de stratégie ; ils constituent, en importance, le troisième centre d'intérêt des auteurs, après les processus et procédures stratégiques (37\%) et les manœuvres stratégiques $(28 \%)$. L'existence d'une revue consacrée à la création et à l'entreprise de petite taille renforce leur place dans le paysage. Toutefois, il convient d'être prudent sur ce constat : il ne s'agit pas, ici, d'évaluer, toutes choses égales par ailleurs, si la PME occupe telle ou telle place. Au contraire, l'existence d'une revue spécifique n'introduit pas un biais, mais constitue un fait marquant, significatif de l'état de maturation

3. Le mot « cocitation » renvoie à une technique statistique qui permet d'écarter, dans les travaux analysés, les citations isolées d'auteurs sans effet sur le développement d'un front de recherche. Pour plus de détails, $c f$. infra 4.2.

4. Sur la notion de structuration d'un champ de recherche, voir Bourdieu (1976), Latour (1989), Déry (1992).

5. Sélection représentative arrêtée en accord avec l'Association internationale de management stratégique, à l'origine de la création de notre base de données (Castagnos, Boissin et Guieu, 1997). 
d'une problématique. S'il existe une revue spécifique, c'est que le champ de recherche est considéré comme important par les acteurs qui y opèrent et interagissent avec d'autres champs (la recherche en stratégie, la recherche en gestion, les pratiques de gestion, de management stratégique ; Déry, 1997).

\subsection{Quelques auteurs dominants}

L'une des principales interrogations, en présence d'un champ de recherche, est celle de savoir qui sont les acteurs agissant (les auteurs) et leur position relative en termes de publication. Soixante-treize auteurs ont contribué à la parution de 60 articles; la production est relativement concentrée avec quatre auteurs réalisant $15 \%$ des contributions.

TABleau 1

Les principaux producteurs francophones d'articles

\begin{tabular}{|c|c|c|}
\hline Nombre d'auteurs & Noms des principaux auteurs & $\begin{array}{l}\text { Nombre d'articles } \\
\text { par auteur }\end{array}$ \\
\hline 1 auteur & Pierre-André Julien & 4 articles \\
\hline 3 auteurs & $\begin{array}{l}\text { Gérald d'Amboise - Bertrand Saporta - } \\
\text { Michel Marchesnay }\end{array}$ & 3 articles \\
\hline 7 auteurs & $\begin{array}{l}\text { Jean-Pierre Bréchet - Jean-Baptiste Carrière - } \\
\text { Alice Guilhon - Elisabeth Lefebvre - } \\
\text { Gilles Paché - Louis Raymond - Gérard Verna }\end{array}$ & 2 articles \\
\hline 62 auteurs & & 1 article \\
\hline
\end{tabular}

La place occupée par la communauté francophone canadienne est très importante et cela est dû en grande partie à la création du centre de recherche sur les PME (Chicha). La spécificité culturelle québécoise - une certaine quête d'autonomie n'est d'ailleurs pas étrangère au développement de la recherche sur les PME, qui jouent un rôle essentiel dans les fondements économiques, linguistiques et sociaux de la province. Les publications des auteurs québécois s'adressent presque exclusivement à la RIPME, qui constitue un support naturel d'expression. Les auteurs français, quant à eux, publient leurs contributions sur les deux continents concernés.

TABLEAU 2

Nations et publications

\begin{tabular}{lcc}
\hline Pays des auteurs des articles & Nombre de contributions & Répartition des contributions \\
\hline France & 47 & $53 \%$ \\
Québec & 36 & $40 \%$ \\
Belgique & 1 & $1 \%$ \\
Autres & 5 & $6 \%$ \\
\hline Total & $\mathbf{8 9}$ & $\mathbf{1 0 0 \%}$ \\
\hline
\end{tabular}

Note : Une contribution est la participation d'un auteur à un article. 


\subsection{Des laboratoires spécialisés}

Trente-cinq laboratoires ont contribué à la publication des 60 articles recensés. La concentration est relativement forte au Québec, où la production est dominée par quatre laboratoires. La recherche française est plus dispersée, hormis cinq centres du Sud de la France. Les collaborations entre centres sont rares, les travaux transfrontaliers quasi inexistants. Néanmoins, c'est à propos de ce thème de recherche que le taux de cosignatures est le plus fort (un sur trois contre un sur cinq pour l'ensemble des publications francophones).

TABLEAU 3

Classement des principaux centres de recherche

\begin{tabular}{lc}
\hline Laboratoire et institution d'enseignement & Nombre de collaborations à article \\
\hline Université du Québec à Trois-Rivières & 14 \\
Université Laval à Québec & 11 \\
ERFI, Université Montpellier I & 6 \\
École polytechnique Montréal & 5 \\
CERAG, ESA Grenoble II & 5 \\
Université Bordeaux I & 4 \\
Université Aix-Marseille II & 4 \\
HEC Montréal & 3 \\
ESC Lyon & 3 \\
\hline
\end{tabular}

\subsection{Une place variable accordée par les revues}

La PME et l'entrepreneuriat sont des thèmes relativement privilégiés par Gestion 2000, la Revue française de gestion, Économies et sociétés et Gestion. En revanche, la REI et PMS n'accorde qu'une place marginale aux écrits sur les firmes de petite taille.

\section{TABLEAU 4}

Revues et place de la PME et de l'entrepreneuriat

\begin{tabular}{lcc}
\hline Revue & $\begin{array}{c}\text { Nombre d'articles parus sur la PME par rapport } \\
\text { aux articles stratégiques }\end{array}$ & Place accordée \\
\hline RFG & 19 sur 109 & $17 \%$ \\
RIPME & 29 sur 32 & $91 \%$ \\
REI & 2 sur 27 & $7 \%$ \\
PMS & 1 sur 26 & $4 \%$ \\
Gestion 2000 & 5 sur 26 & $19 \%$ \\
Gestion & 2 sur 15 & $13 \%$ \\
Économies et sociétés & 2 sur 14 & $14 \%$ \\
\hline TOTAL & $\mathbf{6 0}$ sur 249 & $\mathbf{2 4} \%$ \\
\hline
\end{tabular}


Ainsi, ces différents éléments d'analyse sont autant d'indices de la maturité des champs de la PME et de l'entrepreneuriat : existence d'une revue propre, de laboratoires spécialisés, d'une production scientifique reposant sur des auteurs dominants.

\section{Les thèmes et méthodes de la recherche publiée}

\subsection{Les thèmes privilégiés : processus stratégiques, technologie, dirigeants}

Le poids représenté par les articles consacrés aux PME dans les écrits francophones amène à s'interroger sur le statut de ces organisations dans le champ de la stratégie (Castagnos, Boissin et Guieu, 1997). Grâce aux pratiques de publication des acteurs du champ de recherche, l'analyse des seuls articles consacrés à l'entrepreneuriat et à la PME permet de traiter cette question et de rendre compte des débats internes.

\subsubsection{La création d'entreprise plutôt que l'entrepreneurship}

Vingt contributions sont clairement consacrées à la problématique de l'entrepreneuriat. Les aspects privilégiés sont l'accompagnement, la motivation et la formation des créateurs d'entreprises. Des typologies sont proposées, ainsi que des comparaisons internationales. Assez paradoxalement, les notions de risque et de survie sont peu présentes. De même, l'entrepreneuriat est entendu comme pratique de création. Peu d'articles sont consacrés à l'entrepreneurship, acte d'entreprise, qui constitue le corpus des revues spécialisées Journal of Business Venturing et Entrepreneurship, Theory and Practice. Ce thème est surtout traité au Québec en raison de l'influence américaine, alors qu'en France, pays de tradition centralisée, l'entrepreneur a été plus qu'ailleurs longtemps ignoré.

Les travaux traitant de la spécificité des PME sont disparates : les stratèges s'intéressent à ces organisations dans le cadre de sujets divers, notamment les processus de formation de la stratégie (19 articles), la gestion technologique (10 articles), le fonctionnement des groupes dirigeants ( 7 articles).

\subsubsection{La spécificité des processus et procédures stratégiques}

Deux problématiques dominent la littérature concernant les processus managériaux :

- l'étude de la spécificité des processus décisionnels réalisés en PME, que ce soit en matière d'innovation (Bayad, RIPME, 1993 ; Guilhon, RIPME, 1994), d'internationalisation (Gibiat, RIPME, 1994 ; Lecler, RFG, 1992), de planification stratégique (Mathé et Rivet, RFG, 1993; d'Amboise et Bakanibona, RIPME, 1990);

- l'analyse des contextes de décision, comme la diversité des structures organisationnelles (Desroches, Jog et Taylor, RIPME, 1991) ou le 
changement organisationnel (Julien, Carrière et Raymond, RIPME, 1994 ; Attonaty et Soler, RFG, 1992).

\subsubsection{La gestion de la technologie et les conséquences de l'innovation en PME}

L'acclimatation de la technologie aux PME préoccupe les auteurs francophones:

- la gestion de l'innovation et de la haute technologie représente un premier courant (Guilhon, Guilhon et Péguin, RIPME, 1994 ; Larue de Tournemine, RIPME, 1994);

- les effets induits par la gestion de la technologie, les processus d'apprentissage et de changement organisationnel sont également étudiés (Guilhon, RIPME, 1994 ; Le Bas, RIPME, 1993 ; Julien, Gestion, 1992; Julien, Carrière et Raymond, RIPME, 1994).

\subsubsection{La prépondérance du dirigeant dans les entreprises de petite taille}

Dans le cadre francophone, deux types de préoccupations se font jour :

- l'examen des variables de comportement, telles que la variable culturelle, avec des études de cas nationales (Léone et Le Berre, Économies et sociétés, 1990, pour le Brésil ; Yu, RIPME, 1993, pour la Chine ; Lefebvre, RIPME, 1991, pour le Québec), ou le stade d'évolution de la firme (Lorrain, Belle et Ramangalahy, RIPME, 1994);

- le recensement de types de comportement, en termes financiers (Degos, RFG, 1993), face à l'innovation (Lefebvre, RIPME, 1991), dans ses relations avec son environnement (Chappoz, RIPME, 1991), ou encore en termes de décision stratégique (Mathé et Rivet, RFG, 1993).

\subsubsection{La faible place des manœuvres stratégiques en PME}

Les manœuvres stratégiques réalisées par les PME restent un sujet mineur, représentant six articles, dont seulement deux contributions empiriques. C'est l'étude des stratégies partenariales qui est privilégiée (Fenneteau, RIPME, 1990; Grelaud et Gasse, Gestion 2000, 1995 ; Fernandez et Noël, RIPME, 1993 ; Darréon et Faïçal, RFG, 1993).

\subsection{Des méthodes souvent explicitées}

Les grands traits des méthodes mises en œuvre sont donnés au tableau 5 ; le détail de la grille d'analyse figure à l'annexe II. Les publications consacrées aux PME et à l'entrepreneuriat témoignent d'une préférence des auteurs pour un terrain d'étude, 
notamment en ce qui concerne les articles publiés par la Revue internationale PME. Ainsi, 42 articles sur 60 font référence à un terrain auquel le ou les auteurs se rattachent pour élaborer la connaissance. Pourtant, ces études sont trop souvent réalisées à partir d'observations extérieures réunies par questionnaires sur la base d'un échantillonnage. Les démarches de recherche enracinées dans le terrain (recherche clinique, recherche-action, approche longitudinale, etc.) restent peu fréquentes.

\section{TABLEAU 5}

\section{Caractéristiques des $\mathbf{4 2}$ articles empiriques}

\begin{tabular}{|c|c|c|}
\hline Rapport chercheur-terrain & $\begin{array}{l}\text { extériorité : } 34 \\
\text { étude clinique ou recherche-action : } 8\end{array}$ & \\
\hline \multirow[t]{2}{*}{ Relation terrain-théorie } & Relation forte : 30 articles & Relation faible : 12 articles \\
\hline & $\begin{array}{l}\text { démarche déductive : } 12 \\
\text { démarche inductive : } 18\end{array}$ & $\begin{array}{l}\text { démarche illustrative : } 9 \\
\text { démarche informative : } 3\end{array}$ \\
\hline Temporalité des observations & coupe instantanée : 27 , dont : & étude longitudinale : 11 \\
\hline$\left(\mathrm{nd}^{*}: 4\right)$ & $\begin{array}{l}\text { - coupe instantanée statique : } 22 \\
\text { - coupes instantanées dynamiques : } 5\end{array}$ & \\
\hline $\begin{array}{l}\text { Mode de collecte } \\
\text { des données }\end{array}$ & $\begin{array}{l}\text { cas : } 10 \\
\text { échantillon : } 24 \\
\text { données exhaustives : } 0 \\
\text { sources documentaires : } 7 \\
\text { nd : } 1\end{array}$ & \\
\hline Méthode générique & quantitative : 18 quantitative et quali & qualitative : 15 \\
\hline $\begin{array}{l}\text { Traitement des données } \\
\text { quantitatives }\end{array}$ & $\begin{array}{l}\text { statistiques descriptives : } 8 \\
\text { statistiques explicatives : } 16 \\
\text { nd : } 1\end{array}$ & \\
\hline Visée empirique & $\begin{array}{l}17 \text { travaux exploratoires } \\
14 \text { travaux descriptifs } \\
9 \text { travaux de validation } \\
2 \text { nd }\end{array}$ & \\
\hline
\end{tabular}

* nd signifie non déterminées.

\section{Les bibliographies d'articles}

\subsection{Les principales références citées}

Parmi les principaux auteurs cités, certains sont considérés comme transversaux à tous les thèmes de stratégie, c'est-à-dire qu'on les retrouve également dans les articles consacrés aux manœuvres, aux processus, etc., alors que d'autres sont spécifiques, au sens où ces auteurs occupent une place importante dans les bibliographies des seuls articles consacrés aux PME. 
TABLEAU 6

Principaux auteurs cités

\begin{tabular}{lclc}
\hline Auteurs les plus cités & Nombre de citations & Auteurs les plus cités & Nombre de citations \\
\hline M. Marchesnay & 62 & J. Chicha & 11 \\
P.-A. Julien & 56 & A.C. Cooper & 11 \\
H. Mintzberg & 21 & A.-C. Martinet & 11 \\
M.E. Porter & 20 & E. Lefebvre & 10 \\
R. Robinson & 18 & J.S. Bracker & 9 \\
D. Miller & 17 & Y. Gasse & 9 \\
J.-B. Carrière & 14 & J.M. Toulouse & 9 \\
J.A. Pearce & 13 & L.A. Lefebvre & 8 \\
I. Bamberger & 12 & I. Macmillan & 8 \\
G. d'Amboise & 12 & G. Vozikis & 8 \\
\hline
\end{tabular}

Ainsi, Marchesnay, Julien, Mintzberg, Porter, Miller et Martinet sont-ils largement évoqués par les chercheurs traitant des PME et de l'entrepreneuriat, en plus d'être cités dans d'autres articles de stratégie. En revanche, les autres auteurs du tableau 6 relèvent de la seule problématique de la PME et de l'entrepreneuriat ${ }^{6}$, ils n'ont pas été repérés de manière significative dans les bibliographies d'articles traitant, par exemple, des manœuvres stratégiques ou des processus.

Cette double caractéristique révèle la structuration de la recherche sur la PME : un substrat de référentiels transversaux combiné à un fonds spécifique à la PME. Les deux sources, à savoir la PME et la stratégie, s'interpénètrent et s'enrichissent.

\subsection{La méthode des cocitations et la définition des réseaux bibliographiques}

La citation a plusieurs sens, tant sociaux que cognitifs. La seule analyse des auteurs les plus cités risque de surévaluer les auteurs répertoriés dans les bibliographies bien que non lus, de même que les articles méthodologiques (Callon, Courtial et Penan, 1993). C'est pourquoi la méthode des cocitations est plus fiable que l'analyse des citations pour structurer un champ de recherche. Cette méthode désigne une démarche statistique permettant de repérer les publications majeures (et leurs auteurs) et scientifiquement apparentées en raison de leur présence conjointe dans les bibliographies d'ouvrages ou d'articles publiés postérieurement. Ainsi peuvent être identifiés les travaux et les spécialistes saillants d'une discipline ou d'un thème

6. Une seconde distinction peut être faite entre les articles traitant des PME et ceux portant sur l'entrepreneurship ; dans ces derniers, outre les auteurs communs aux deux champs, apparaît notamment Gartner. 
de recherche (éléments et acteurs au cœur de la recherche), les phases chronologiques de développement des connaissances, l'intensité des associations et les éventuelles ramifications entre fronts de recherche. L'analyse des cocitations multiples suppose donc que les auteurs francophones emploient ensemble, à plusieurs reprises, le même couple de références. Cette cocitation ne relève pas du simple hasard, mais de la complémentarité de deux textes, de deux auteurs, dans le raisonnement des chercheurs utilisateurs. Finalement, le traitement des bibliographies, comparable à un monde vivant, s'apparente à l'approche de l'organisme du biologiste François Jacob (1970, p. 14) : «l'organisme n'est pas dissociable en ses constituants, mais il y a souvent intérêt à le regarder comme l'élément d'un système d'ordre supérieur, groupe, espèce, population, famille écologique ». La procédure utilisée pour relever les cocitations et construire les réseaux d'auteurs figure à l'annexe III.

Des réseaux sont donc constitués. Notons au passage que le mot réseau désigne une cartographie des auteurs (et de leurs publications) les plus fréquemment associés à un domaine de recherches (une discipline, un thème, etc.). Si bien que tout réseau comporte deux catégories d'associations d'auteurs. La première (en caractère gras au schéma 1 et suivants) est composée de paires d'auteurs cocités dans les bibliographies et se révèle représentative du cœur de la recherche (c'est-àdire du thème ou du sujet étudié); les auteurs concernés sont qualifiés d'internes au champ de recherche (ou, plus précisément, au thème ou à toute autre subdivision disciplinaire identifiée). La seconde catégorie (auteurs mentionnés en style normal au schéma 1 et suivants) résulte d'associations d'auteurs issus d'autres réseaux; ces auteurs à multisollicitations bibliographiques de leurs travaux sont dits externes à la subdivision disciplinaire analysée (chacun de ces auteurs occupe souvent le premier rang au sein d'un autre réseau) : ils constituent des « ponts » interréseaux.

L'analyse des réseaux d'auteurs constitutifs de l'ensemble des articles francophones de stratégie (voir schéma 1) a été publiée (Boissin, Castagnos et Guieu, 1999). On se bornera donc, ici, à examiner les seuls réseaux traitant des PME et de l'entrepreneuriat, qui sont au nombre de quatre.

\subsection{L'identification des réseaux bibliographiques}

Parmi les 7075 références citées dans les bibliographies des 249 articles francophones en stratégie publiés entre 1990 et 1995 , quatre réseaux sont identifiés comme relevant explicitement des problématiques des PME et de l'entrepreneuriat (tableau 7). Deux d'entre eux sont qualifiés d'autocentrés, puisque chacun ne recouvre qu'un seul thème : identité de la PME et entrepreneuriat. L'analyse laisse également apparaître deux réseaux-carrefours, dont l'objet est de traiter d'un thème de gestion stratégique dans le contexte de la PME : l'innovation et la planification. Ces quatre réseaux sont examinés tour à tour dans cette section. Si la forme réticulaire de la présentation fait bien ressortir la proximité intellectuelle des auteurs d'un même 
réseau, elle ne nous en donne pas le sens. Aussi procédera-t-on à l'analyse détaillée des quatre réseaux qui structurent la recherche francophone en stratégie avec, pour chacun d'entre eux, une présentation :

- du thème traité et des implications théoriques éventuelles ;

- des apports essentiels des principaux auteurs et écoles de recherche;

- des articles s'inspirant des auteurs mis en réseau, ainsi que la raison de cette mobilisation.

\section{SCHÉMA 1}

\section{Représentation des bibliographies de la recherche francophone en stratégie}

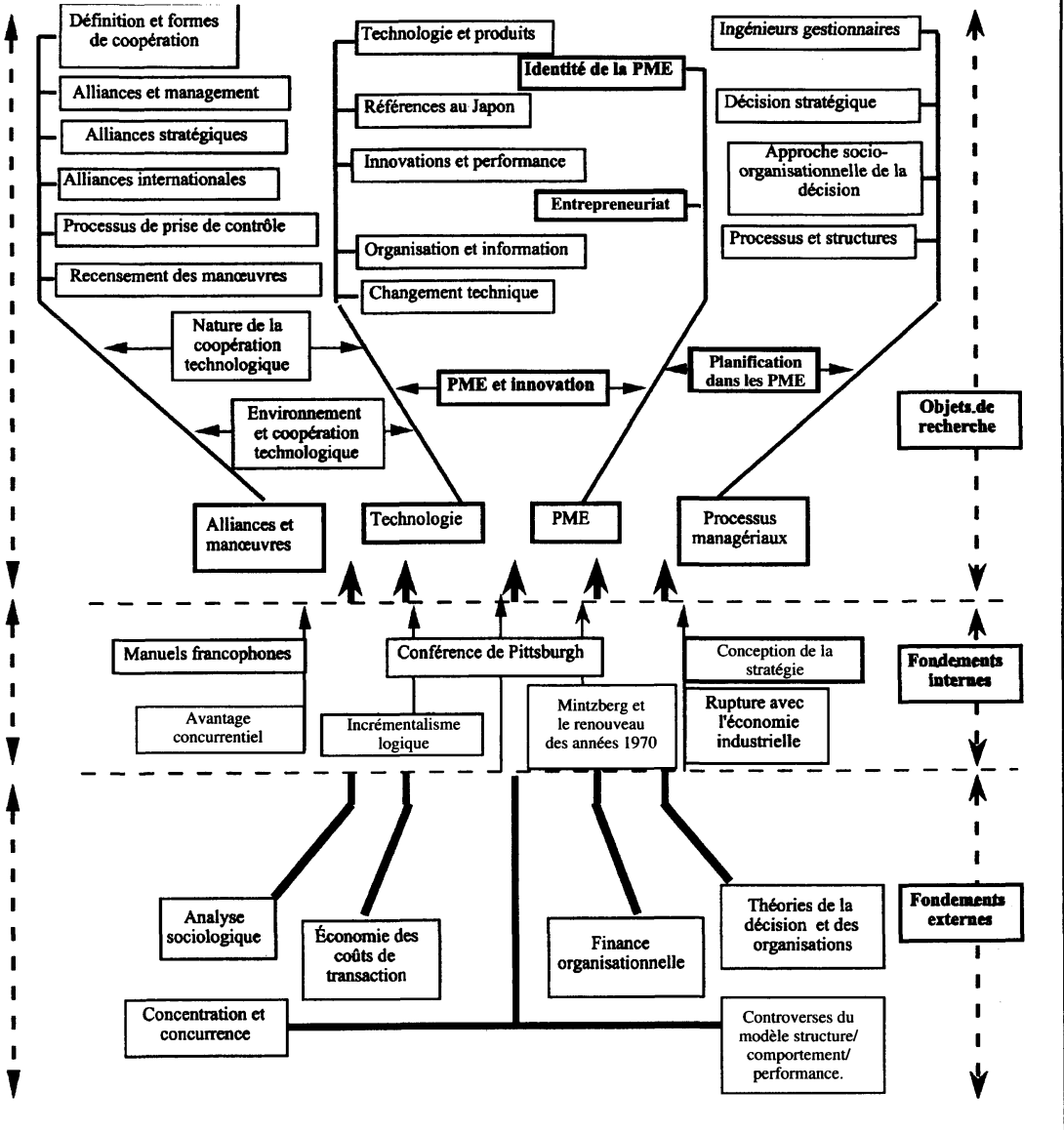




\section{TABleau 7}

Structuration des sujets d'étude en réseaux autocentrés et carrefours

\begin{tabular}{ll}
\hline Réseaux autocentrés & Réseaux carrefours \\
\hline - Identité de la PME & - Planification dans les PME \\
(Chicha-Julien-Marchesnay) & (Robinson et d'Amboise) \\
- Entrepreneuriat (Cooper) & $-\begin{array}{l}\text { PME et innovation } \\
\text { (Toulouse et Larue de Tournemine) }\end{array}$ \\
\hline 2 réseaux propres & 2 réseaux-carrefours \\
\hline
\end{tabular}

\subsubsection{La spécificité «polymorphe » de la PME}

Le premier réseau, fréquemment cité, a trait à la définition de l'objet d'étude; ce réseau gravite autour de P.-A. Julien. Les auteurs francophones associés sont Chicha, en tant qu'initiateur historique ; les autres tenants de l'école québécoise de la PME, Hébert, Jacob, Carrière et Filion ; Marchesnay, partenaire français de la recherche sur la PME en général et la «très petite entreprise » (TPE) en particulier. Goldhar et Jelinek sont les seuls auteurs anglophones du réseau.

SCHÉMA 2

Réseau «Identité de la PME »

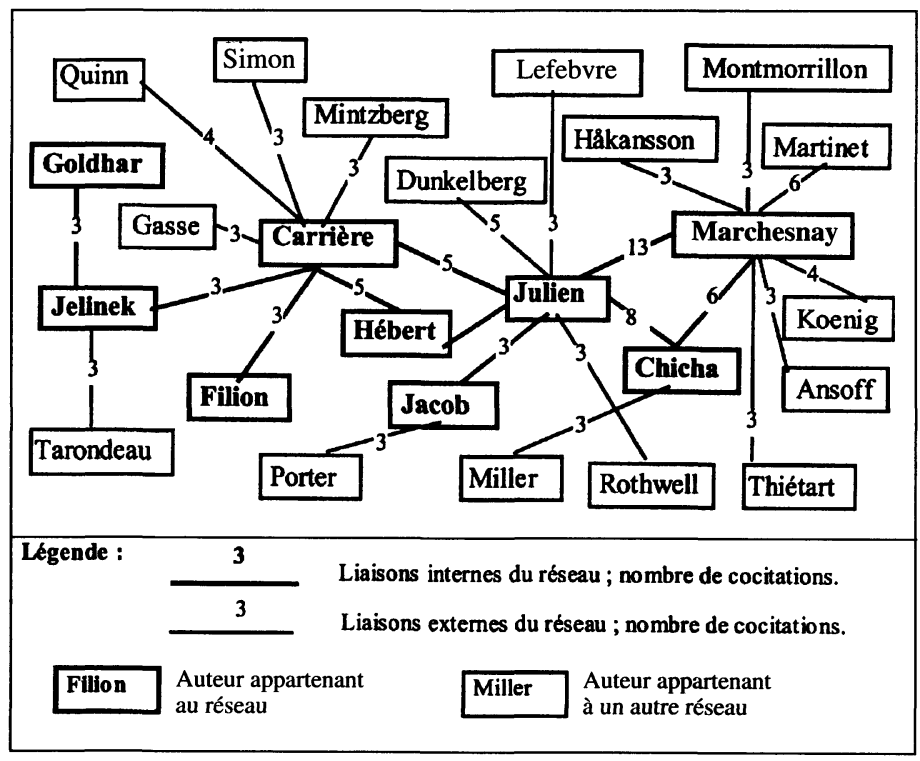


Julien et Marchesnay sont fortement présents dans les bibliographies d'articles. Leurs travaux ont essentiellement consisté à développer l'étude, sous toutes ses formes et dans de nombreuses directions, de l'objet apparemment spécifique, mais divers, de la PME. Par exemple, Marchesnay étudie tour à tour le marketing de la petite entreprise (RIPME, 1988), l'externalisation de services (Revue de l'économie méridionale, 1988), la petite entreprise comme un espace de transaction (Marchesnay, Entrepreneurial and Regional Development, 1990). Insistant sur la place déterminante du PDG, il introduit également une typologie (les PIC et les CAP) de dirigeants de PME, qui a été par la suite reprise et testée.

La collaboration entre Marchesnay et Julien est fréquente. Les travaux de clarification de la spécificité de la PME, publiés dans des manuels (Julien et Marchesnay, La petite entreprise, 1988), comme ceux retenant une définition multicritère des PME (Julien, RIPME, 1990) ou appelant au passage des procédures aux processus stratégiques (Julien et Marchesnay, PMS, 1992) sont rapidement intégrés dans les bibliographies. Dans leurs écrits, on retrouve une PME «idéaltypée », centrée sur le dirigeant. De ce tronc commun, de multiples champs d'étude s'ouvrent en PME.

Les autres auteurs du réseau sont plus spécialisés :

- Hébert et Carrière, associés à Julien au sein du GREPME, fournissent un meilleur éclairage de la mise en place des nouvelles technologies en milieu PME, par des enquêtes réalisées au Québec sur de vastes échantillons (Julien et Hébert, Journal of Small Business and Entrepreneurship, 1986 ; Carrière et Julien, Rapport, 1992 ; Julien, Carrière et Hébert, RIPME, 1988). Jacob, également intéressé par l'introduction de technologies dans les PME, insiste pour sa part sur l'adoption et le renouvellement accéléré des technologies dans les nouvelles formes organisationnelles adoptées par les PME (Jacob, GREPME, 1993);

- Filion développe la notion de vision stratégique du dirigeant de PME, susceptible d'être un facteur clé de réussite (Gestion, 1989). Testant l'hypothèse d'association positive entre la manifestation de la vision stratégique et la performance, Nikongolo-Bakenda, d'Amboise et Garnier, concluront à la nécessité d'une meilleure définition (RIPME, 1994);

- Goldhar et Jelinek ont analysé les implications organisationnelles, économiques et stratégiques de la fabrication flexible informatisée (Interfaces, 1985) en centrant leurs résultats sur la notion d'investissement intellectuel.

Les auteurs du réseau « identité de la PME » sont utilisés dans les bibliographies lorsqu'il s'agit de traiter du rôle de l'investissement intellectuel dans l'implantation de nouvelles techniques en PME (Guilhon, RIPME, 1994), d'analyser le passage des procédures de planification à des processus stratégiques (Julien et 
Marchesnay, PMS, 1992), d'évaluer le profil distinctif des dirigeants de PME innovatrices (Lefebvre, RIPME, 1991), de commenter l'introduction de nouvelles technologies dans les PME manufacturières québécoises (Julien, Gestion, 1992), d'étudier les comportements d'intégration marketing - stratégie dans les PME (Saporta et Lapousse Madrid, RIPME, 1995) ou encore de juger de la compatibilité pour les PME de l'innovation et de l'exportation (Guilhon, Guilhon et Péguin, RIPME, 1994). Cette diversité de thèmes montre que les auteurs citants «s'alimentent» en fondements conceptuels auprès des auteurs du réseau «identité de la PME ».

\subsubsection{L'entrepreneuriat}

La représentation de l'entrepreneuriat dans la littérature francophone en stratégie est singulière. En effet, aucun auteur du réseau découlant de la base de données francophone n'apparaît dans le palmarès de citations établi par Béchard à partir des bibliographies des articles parus dans trois revues américaines en entrepreneurship (Entrepreneurship, Theory and Practice, Journal of Business Venturing, Journal of Small Business Management). Pourtant, les travaux cités sur l'entrepreneurship

SCHÉMA 3

Réseau « entrepreneuriat»

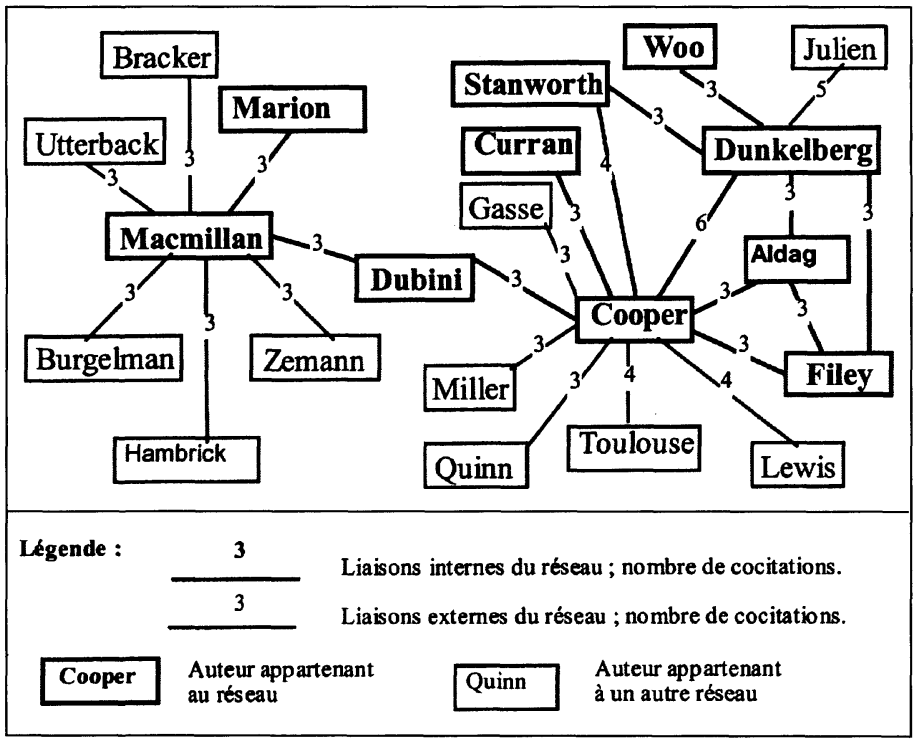


dans les revues francophones sont majoritairement anglophones. MacMillan et Dubini s'intéressent aux démarrages d'activité. Woo, Cooper et Dunkelberg proposent des typologies entrepreneuriales (Journal of Business Venturing, 1991), après que Stanworth et al. eurent montré « qui devient entrepreneur » (International Small Business Journal, 1989). En outre, les grands auteurs historiques, à commencer par Schumpeter, sont peu présents.

Le réseau identifié provient des bibliographies de trois types de publications :

- les écrits relevant de l'étude des caractéristiques clés de l'entrepreneurship et s'inspirant de toute la palette des auteurs du réseau. Capiez (RIPME, 1992) propose un diagnostic d'émergence pour établir les chances de succès des petites entreprises. Il en va de même avec Livian et Marion (RIPME, 1991) pour la création d'entreprise. Bruyat, quant à lui, propose un cadre épistémologique de réflexion de l'entrepreneurship (RFG, 1994);

- la littérature sur le type de dirigeant (Lorrain, Belle et Ramangahaly, RIPME, 1994 ; Blais et Toulouse, RIPME, 1990);

- des synthèses thématiques de la définition de la PME (Julien, RIPME, 1990) ou de la création d'entreprise (Saporta, RFG, 1994) mobilisant de façon inégale divers auteurs du réseau.

\subsubsection{La planification et la performance dans les PME}

Le thème de la planification stratégique est surtout abordé par d'Amboise et ses collaborateurs de l'Université Laval à Québec. Cet important courant de recherche a su renouveler le thème en intégrant des processus de planification plus managériaux, moins finalisés et formalisés, et en testant la notion de vision. Le bilan est contrasté : le lien entre planification et performance est distendu dans le cas de la PME (RIPME, 1990), de même que la relation entre vision et performance.

Les auteurs du réseau se ventilent en quatre sous-groupes. On retrouve, tout d'abord, d'Amboise et Gasse pour leurs travaux respectifs sur les pratiques de management des PME ; puis, les tenants anglophones de l'étude de la planification (Venkatraman et Ramanujam, 1986), qui fourniront des indicateurs de performance ; ensuite, les travaux sur la planification et la performance au cours des étapes de développement de la firme de petite taille (Robinson, Pearce, Mescon et Vozikis, 1984); enfin, les études sur la relation planification-performance dans le contexte des PME (Ackelsberg et Arlow, 1985 ; Pearce et Robinson, 1979-1987 ; d'Amboise, 1989, 1990). Ces dernières aboutiront à montrer le paradoxe de vouloir formaliser une organisation à caractère entrepreneurial alors qu'elle tire sa force de la flexibilité. 
SCHÉMA 4

Réseau «Planification dans les PME et performance»

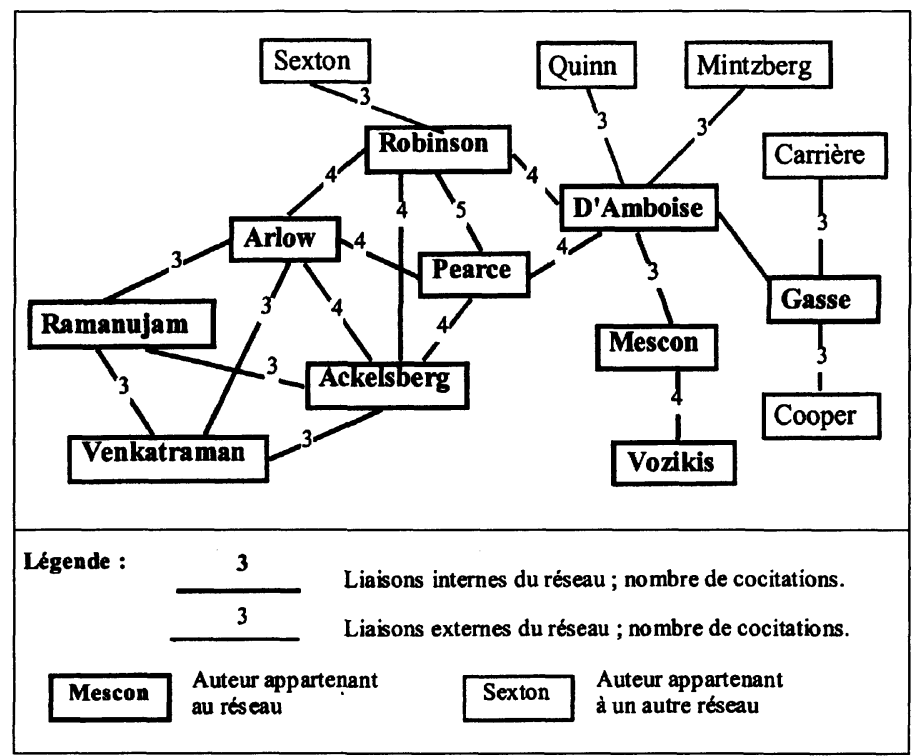

Deux articles analysés sont représentatifs de la tentative de synthèse faite dans les années 1990-1995 par cette école ; ils sont révélateurs de l'amendement de la notion de planification proposé par les auteurs. Un premier article de synthèse est publié en 1990 par d'Amboise et Bakanibona et fournit un bilan contrasté : c'est moins le temps consacré à l'activité que la qualité de la planification qui détermine la performance. Un second sera publié en 1994, par Nikongolo-Bakenda, d'Amboise et Garnier, qui cherchera à mettre en évidence «l'association entre la manifestation de la vision stratégique des propriétaires-dirigeants de PME et la performance de leur entreprise ». L'écart entre les deux articles introduit la notion de vision adjointe aux études sur les procédures. En 1992, la transition aura été clairement affirmée par Julien et Marchesnay, qui font passer la stratégie des PME « des procédures aux processus ». Les modalités de management de l'entreprise de petite taille ne relèvent plus des modèles de planification : elles réclament des modalités de mesure de la performance plus adaptées. Un triple mouvement est alors engagé : un désintérêt relatif pour la notion de performance, le discrédit du terme de «planification », la difficulté d'étudier la performance dans un contexte managérial moins formalisé. 


\subsubsection{La PME et l'innovation}

La connexion entre innovation et entreprise de petite taille est "naturelle», Schumpeter ayant défini «l'essence de l'entrepreneuriat [comme] la perception et l'exploitation de nouvelles opportunités [...] » (Schumpeter, 1928, cité par Filion, 1997, p. 134).

\section{SCHÉMA 5}

Réseau «PME et innovation »

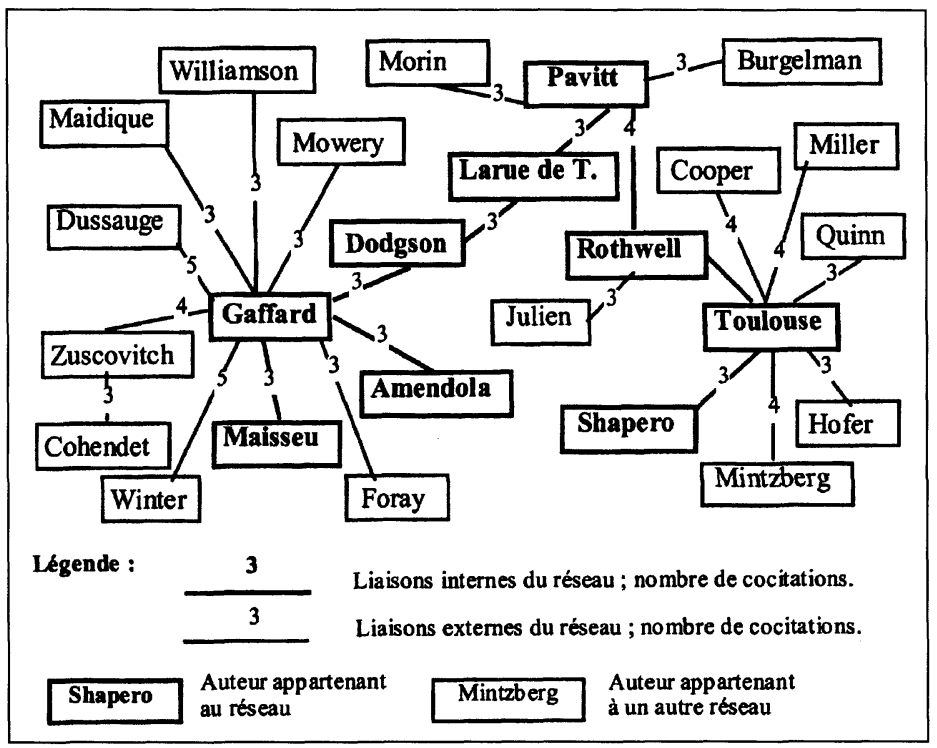

Le réseau sur l'innovation regroupe une grande variété d'auteurs qui s'agrègent en deux catégories : la première place la structure au centre de l'analyse de l'innovation; la seconde s'intéresse à l'homme porteur d'innovation.

- La technologie et l'innovation sont d'abord conçues comme des moteurs pour les PME, comme un support de la structure et un facteur de changement. L'analyse est centrée sur l'organisation qui est gestionnaire de l'innovation. Ainsi, Larue de Tournemine (RIPME, 1994), pour décrire la complémentarité entre grande entreprise et PME en matière d'innovation technologique, ou encore Le Bas (RIPME, 1993), pour décrire le comportement d'investissement en recherche technologique des moyennes entreprises industrielles, empruntent à Gaffard (1990), une économie industrielle de l'innovation, à Dodgson (1991), une théorie de l'innovation comme un apprentissage technologique, à Pavitt (1984), sa taxonomie des trajectoires 
technologiques qui varient selon l'intensité de la recherche-développement de la firme et le type de produits conçus;

- L'entrepreneur-innovateur constitue le second pôle d'étude. La dimension sociale et psychologique de l'entrepreneur est abordée par les auteurs fondamentaux. Le comportement innovateur trouve sa source dans le désir d'évasion et de disposer d'un champ de contrôle suffisant (Shapero, 1975, 1982). Les travaux de Rothwell (1978), également cités, soulignent la forte intensité innovatrice des firmes les plus petites. Fayolle (RFG, 1994) étudie la trajectoire de l'ingénieur-entrepreneur. Lefebvre (RIPME, 1991) traite des profils distinctifs des dirigeants innovateurs. Blais et Toulouse (RIPME, 1990) présentent les motivations des entrepreneurs. Bayad (RIPME, 1993) insiste sur la prépondérance des moyens humains dans l'effort de recherche.

\section{Conclusion}

Cet article propose une structuration de la recherche en stratégie de la PME en explicitant la constitution du champ sous ses aspects sociaux (relations entre membres de la communauté) et cognitifs (filiations et connexions intellectuelles entre membres de la communauté). De surcroît, cette recherche sur le contenu des articles francophones et leurs bibliographies débouche sur une représentation historique. Déry (1996) propose une structuration historique du champ de la stratégie en six étapes ; ces subdivisions sont reprises ici. Le nombre important de références datant des périodes dites d'institutionnalisation (1980-1984) et d'objectivation (1985-1995) de la stratégie révèle une intégration rapide des références récentes, signe d'une communauté dynamique (tableau 8).

TABLEAU 8

Historicité des références bibliographiques

\begin{tabular}{lccccccc}
\hline & $\begin{array}{l}\text { Incubation } \\
\text { 1900-1959 }\end{array}$ & $\begin{array}{c}\text { Émergence } \\
\text { 1960-1969 }\end{array}$ & $\begin{array}{c}\text { Croissance } \\
\text { 1970-1979 }\end{array}$ & $\begin{array}{c}\text { Institutionnalisation } \\
\mathbf{1 9 8 0 - 1 9 8 4}\end{array}$ & $\begin{array}{c}\text { Objectivation } \\
\text { 1985-1989 }\end{array}$ & $\begin{array}{c}\text { Objectivation } \\
\mathbf{1 9 9 0 - 1 9 9 6}\end{array}$ & Total \\
\hline $\begin{array}{l}\text { Références } \\
\text { par période }\end{array}$ & 13 & 47 & 172 & 265 & 630 & 377 & 1504 \\
$\begin{array}{l}\text { Répartition } \\
\text { des références }\end{array}$ & $0,9 \%$ & $3,1 \%$ & $11,4 \%$ & $17,6 \%$ & $41,9 \%$ & $25,1 \%$ & $100 \%$ \\
\hline
\end{tabular}

Le positionnement historique des quatre réseaux de cocitations identifiés met en évidence la périodicité des fronts de recherche stratégiques sur la PME (tableau 9). Ainsi, le thème de la planification a-t-il été très important au cours de la dernière décennie, pour n'être plus au cœur des débats dès les années 1990. En revanche, l'innovation et l'identité des PME constituent des questions de recherche très 
discutées au cours des périodes les plus récentes. L'entrepreneuriat, au parcours historique atypique dans les bibliographies recensées, pourrait, à partir de la seconde moitié de la décennie actuelle, devenir un thème clé de recherches, aussi bien sur la notion d'entreprise que sur celle de la diversité des entrepreneurs (Marchesnay, 1998).

\section{Tableau 9}

\section{Structuration historique des réseaux de cocitations PME}

\begin{tabular}{|c|c|c|c|c|c|c|}
\hline $\begin{array}{l}\text { Réseaux et nombre de } \\
\text { références des auteurs } \\
\text { du réseau par période }\end{array}$ & $\begin{array}{l}\text { Incubation } \\
1900-1959\end{array}$ & $\begin{array}{l}\text { Émergence } \\
1960-1969\end{array}$ & $\begin{array}{l}\text { Croissance } \\
1970-1979\end{array}$ & $\begin{array}{c}\text { Institutionnalisation } \\
1980-1984\end{array}$ & $\begin{array}{c}\text { Objectivation } \\
1985-1989\end{array}$ & $\begin{array}{c}\text { Objectivation } \\
1990-1996\end{array}$ \\
\hline Entrepreneuriat & & & 10 & 9 & 18 & 7 \\
\hline PME et innovation & & & 4 & 10 & 29 & 30 \\
\hline Identité de la PME & & & 3 & 13 & 80 & 73 \\
\hline $\begin{array}{l}\text { Planification } \\
\text { dans les PME }\end{array}$ & & & & 33 & 34 & 6 \\
\hline
\end{tabular}

Ces développements sont susceptibles d'alimenter les interrogations actuelles sur la constitution du champ de recherche, notamment par le rappel du contexte historique présidant aux débats autour de la spécificité de la PME.

\section{Annexe I}

\section{Liste des articles francophones publiés entre 1990 et 1995, portant sur les PME ou sur l'entrepreneuriat}

\begin{tabular}{|c|c|c|}
\hline Auteurs & Titre de l'article & Références complètes \\
\hline N.G. Leone et M. Le Berre & $\begin{array}{l}\text { Une typologie des dirigeants de PME : l'exemple } \\
\text { des dirigeants de PME du Nordeste brésilien }\end{array}$ & $\begin{array}{l}\text { Économies et sociétés - série SG, } \\
1990, \mathrm{n}^{\circ} 15, \text { p. } 7-37 .\end{array}$ \\
\hline J. Lebraty & Management et gestion : quel apprentissage & $\begin{array}{l}\text { Économies et sociétés, } 1992, \mathrm{SG} \\
\mathrm{n}^{0} 18, \text { p. } 131-159 .\end{array}$ \\
\hline Z. Su & $\begin{array}{l}\text { Le développement de l'entrepreneurship privé en } \\
\text { Chine : caractéristiques et problèmes }\end{array}$ & $\begin{array}{l}\text { Gestion } 2000,1993, \text { vol. } 9, \mathrm{n}^{\circ} 2 \\
\text { p. } 103-117 .\end{array}$ \\
\hline G. d'Amboise et G. Verna & De l'entrepreneur à l'intrapreneur & $\begin{array}{l}\text { Gestion 2000, 1993, vol. } 9, \mathrm{n}^{0} 2 \text {, } \\
\text { p. } 13-30 \text {. }\end{array}$ \\
\hline J.-J. Obrecht & L'éthique et le nouvel entrepreneur & $\begin{array}{l}\text { Gestion } 2000,1994, \text { vol. } 10, \mathrm{n}^{\circ} 1 \text {, } \\
\text { p. } 37-55 .\end{array}$ \\
\hline G. Verna & $\begin{array}{l}\text { Croissance ou survie ? Quelle sera l'attitude la } \\
\text { plus probable pour une petite entreprise légale } \\
\text { du tiers monde? }\end{array}$ & $\begin{array}{l}\text { Gestion } 2000,1994 \text {, vol. } 10, \mathrm{n}^{\circ} 4 \text {, } \\
\text { p. } 93-113 \text {. }\end{array}$ \\
\hline J. Grelaud et Y. Gasse & $\begin{array}{l}\text { Processus de réalisation des alliances stratégiques } \\
\text { entre PME occidentales et PME sénégalaises. }\end{array}$ & $\begin{array}{l}\text { Gestion } 2000,1995 \text {, vol. } 11, \mathrm{n}^{0} 4 \text {, } \\
\text { p. } 89-107\end{array}$ \\
\hline $\begin{array}{l}\text { E. Lefebvre, L. Lefebvre } \\
\text { et R. Poupart }\end{array}$ & Innovation et PME: les enjeux stratégiques & $\begin{array}{l}\text { Gestion, 1991, vol. } 16, \mathrm{n}^{\circ} 2 \text {, } \\
\text { p. } 32-37\end{array}$ \\
\hline
\end{tabular}




\section{Annexe I (suite)}

\begin{tabular}{|c|c|c|}
\hline Auteurs & Titre de l'article & Références complètes \\
\hline P.-A. Julien & $\begin{array}{l}\text { Les nouvelles technologies dans les PME } \\
\text { manufacturières québécoises }\end{array}$ & $\begin{array}{l}\text { Gestion, 1992, vol. 17, no } 4 \text {, } \\
\text { p. 29-38 }\end{array}$ \\
\hline $\begin{array}{l}\text { P.-A. Julien et } \\
\text { M. Marchesnay }\end{array}$ & $\begin{array}{l}\text { Des procédures aux processus stratégiques } \\
\text { en PME }\end{array}$ & PMS, 1992, vol. 1, p. 97-129. \\
\hline G. Paché & $\begin{array}{l}\text { L'impact des stratégies d'entreprise } \\
\text { sur l'organisation industrielle: PME et réseaux } \\
\text { de compétences }\end{array}$ & REI, $1991, n^{0} 56$, p. $58-70$. \\
\hline P. Mustar & $\begin{array}{l}\text { Organisations, technologies et marchés en } \\
\text { création : la genèse des PME high-tech }\end{array}$ & REI, $1994, n^{0} 67$, p. $156-174$. \\
\hline $\begin{array}{l}\text { G. d'Amboise et } \\
\text { A. Bakanibona }\end{array}$ & $\begin{array}{l}\text { La planification dans les PME: une synthèse } \\
\text { de résultats empiriques. Conclusions } \\
\text { et recommandations }\end{array}$ & $\begin{array}{l}\text { Revue internationale PME, } 1990, \\
\text { vol. } 3, \mathrm{n}^{\circ} 2, \text { p. } 147-166 .\end{array}$ \\
\hline H. Fenneteau & $\begin{array}{l}\text { Mise en concurrence des fournisseurs } \\
\text { ou partenariat? }\end{array}$ & $\begin{array}{l}\text { Revue internationale PME, } 1990, \\
\text { vol. } 3, \mathrm{n}^{\circ} 2, \text { p. } 167-192 .\end{array}$ \\
\hline R. Blais et J.M. Toulouse & $\begin{array}{l}\text { Les motivations des entrepreneurs : une étude } \\
\text { empirique de } 2278 \text { fondateurs d'entreprises } \\
\text { dans } 14 \text { pays }\end{array}$ & $\begin{array}{l}\text { Revue internationale PME, } 1990, \\
\text { vol. } 3, \mathrm{n}^{\mathrm{s}} 3-4, \mathrm{p} .269-300 .\end{array}$ \\
\hline J.-B. Carrière & $\begin{array}{l}\text { La vision stratégique en contexte de PME: } \\
\text { cadre théorique et étude empirique }\end{array}$ & $\begin{array}{l}\text { Revue internationale PME, } 1990, \\
\text { vol. } 3, \mathrm{n}^{\mathrm{s}} 3-4, \mathrm{p} .301-326 .\end{array}$ \\
\hline P.-A. Julien & Vers une typologie multicritère des PME & $\begin{array}{l}\text { Revue internationale PME, } 1990 \text {, } \\
\text { vol. } 3, \mathrm{n}^{\mathrm{os}} 3-4, \text { p. } 411-425 .\end{array}$ \\
\hline $\begin{array}{l}\text { H. Frank, G. Plaschka, } \\
\text { D. Roessl et H. Welsch }\end{array}$ & $\begin{array}{l}\text { Valeurs guidant la planification des nouvelles } \\
\text { entreprises: une comparaison internationale }\end{array}$ & $\begin{array}{l}\text { Revue internationale PME, } 1991 \text {, } \\
\text { vol. } 4, \mathrm{n}^{0} 1, \text { p. } 5-27 .\end{array}$ \\
\hline Y.F. Livian et S. Marion & $\begin{array}{l}\text { De l'évaluation des projets de création } \\
\text { d'entreprises au pronostic du succès }\end{array}$ & $\begin{array}{l}\text { Revue internationale PME, 1991, } \\
\text { vol. } 4, \mathrm{n}^{0} 1, \mathrm{p} .107-129 .\end{array}$ \\
\hline M. Marchesnay & $\begin{array}{l}\text { Mintzberg on PME. Apropos d'un récent } \\
\text { ouvrage de Henri Mintzberg }\end{array}$ & $\begin{array}{l}\text { Revue internationale PME, 1991, } \\
\text { vol. } 4, \mathrm{n}^{0} 1, \text { p. 131-138. }\end{array}$ \\
\hline E. Lefebvre & Profil distinctif des dirigeants de PME innovatrices & $\begin{array}{l}\text { Revue internationale PME, 1991, } \\
\text { vol. } 4, \mathrm{n}^{0} 3, \text { p. } 7-26 .\end{array}$ \\
\hline $\begin{array}{l}\text { J. Desroches, V. Jog } \\
\text { et W. Taylor }\end{array}$ & $\begin{array}{l}\text { Changements de la structure et de la dynamique } \\
\text { managériale dans les entreprises devenues publiques }\end{array}$ & $\begin{array}{l}\text { Revue internationale PME, 1991, } \\
\text { vol. } 4, \mathrm{n}^{\circ} 3 \text {, p. } 27-51 \text {. }\end{array}$ \\
\hline Y. Chappoz & $\begin{array}{l}\text { La gestion de l'interactivité } \\
\text { entreprise/environnement }\end{array}$ & $\begin{array}{l}\text { Revue internationale PME, 1991, } \\
\text { vol. } 4, \mathrm{n}^{\circ} 3 \text {, p. 53-75. }\end{array}$ \\
\hline A. Capiez & $\begin{array}{l}\text { Les chances de succès des petites entreprises: } \\
\text { vers un diagnostic d'émergence. }\end{array}$ & $\begin{array}{l}\text { Revue internationale PME, 1992, } \\
\text { vol. } 5, \mathrm{n}^{\circ} 2, \text { p. 103-132. }\end{array}$ \\
\hline I. Géniaux & Les entreprises de développement technologique & $\begin{array}{l}\text { Revue internationale PME, 1993, } \\
\text { vol. } 6, \mathrm{n}^{\mathrm{os}} 3-4, \text { p. } 37-58 .\end{array}$ \\
\hline G. Fernandez et A. Noël & PME, mondialisation et stratégies & $\begin{array}{l}\text { Revue internationale PME, 1993, } \\
\text { vol. } 6, \mathrm{n}^{\mathrm{os}} 3-4, \text { p. } 145-163 .\end{array}$ \\
\hline P. Robic & $\begin{array}{l}\text { Une nouvelle mesure de la stratégie } \\
\text { de diversification des PME }\end{array}$ & $\begin{array}{l}\text { Revue internationale PME, 1993, } \\
\text { vol. } 6, \mathrm{n}^{\mathrm{os}} 3-4, \text { p. } 9-35 .\end{array}$ \\
\hline H. Yu & $\begin{array}{l}\text { Décision d'investissement des petites entreprises } \\
\text { chinoises dans un système de responsabilité }\end{array}$ & $\begin{array}{l}\text { Revue internationale PME, 1993, } \\
\text { vol. } 6, \mathrm{n}^{\circ} 1, \text { p. 11-27. }\end{array}$ \\
\hline M. Bayad & $\begin{array}{l}\text { L'effort de recherche de la PME industrielle } \\
\text { face au développement des produits nouveaux: } \\
\text { la prépondérance des moyens humains }\end{array}$ & $\begin{array}{l}\text { Revue internationale PME, 1993, } \\
\text { vol. } 6, \mathrm{n}^{\circ} 1, \text { p. } 29-48 .\end{array}$ \\
\hline H. Lesca et L. Raymond & $\begin{array}{l}\text { Expérimentation d'un système expert pour } \\
\text { l'évaluation de la veille stratégique dans les PME }\end{array}$ & $\begin{array}{l}\text { Revue internationale PME, 1993, } \\
\text { vol. } 6, \mathrm{n}^{\circ} 1, \text { p. } 49-65 .\end{array}$ \\
\hline C. Le Bas & $\begin{array}{l}\text { Le comportement d'investissement en ressources } \\
\text { technologiques externes et l'absorption technolo- } \\
\text { gique dans les moyennes entreprises industrielles }\end{array}$ & $\begin{array}{l}\text { Revue internationale PME, 1993, } \\
\text { vol. } 6, \mathrm{n}^{\circ} 2 \text {, p. } 35-64 .\end{array}$ \\
\hline $\begin{array}{l}\text { J.M. Nikongolo-Bakenda, } \\
\text { G. d'Amboise } \\
\text { et B. Garneir }\end{array}$ & $\begin{array}{l}\text { L'association entre la manifestation de la vision } \\
\text { stratégique des propriétaires-dirigeants de PME } \\
\text { et la performance de leur entreprise }\end{array}$ & $\begin{array}{l}\text { Revue internationale PME, 1994, } \\
\text { vol. } 7, \mathrm{n}^{\circ} 1, \text { p. } 35-61 .\end{array}$ \\
\hline
\end{tabular}




\section{Annexe I (suite)}

\begin{tabular}{|c|c|c|}
\hline Auteurs & Titre de l'article & Références complètes \\
\hline $\begin{array}{l}\text { J. Lorrain, A. Belle } \\
\text { et C. Ramangalahy }\end{array}$ & $\begin{array}{l}\text { Relation entre le profil de comportement des } \\
\text { propriétaires-dirigeants et le stade d'évolution } \\
\text { de leur entreprise }\end{array}$ & $\begin{array}{l}\text { Revue internationale PME, } \\
1994, \text { vol. } 7, \mathrm{n}^{0} 1, \text { p. } 9-34 .\end{array}$ \\
\hline M. Gibiat & $\begin{array}{l}\text { Les modèles intégrés de la décision d'exporter en } \\
\text { PME / PMI : synthèse des recherches depuis } 20 \text { ans }\end{array}$ & $\begin{array}{l}\text { Revue internationale } P M E \text {, } \\
1994, \text { vol. } 7, \mathrm{n}^{\circ} 2 \text {, p. 11-29. }\end{array}$ \\
\hline $\begin{array}{l}\text { A. Guilhon, B. Guilhon } \\
\text { et D. Peguin }\end{array}$ & $\begin{array}{l}\text { Innovation et exportation : quelle compatibilité } \\
\text { pour les PME? }\end{array}$ & $\begin{array}{l}\text { Revue internationale PME, } \\
1994, \text { vol. } 7, \mathrm{n}^{\circ} 2 \text {, p. 85-104. }\end{array}$ \\
\hline A. Guilhon & $\begin{array}{l}\text { Le rôle de l'investissement intellectuel dans la } \\
\text { stratégie d'implantation des nouvelles techniques } \\
\text { dans les PME }\end{array}$ & $\begin{array}{l}\text { Revue internationale PME, } \\
1994, \text { vol. } 7, \mathrm{n}^{\circ \mathrm{s}} 3-4, \text { p. } 147-171 .\end{array}$ \\
\hline R. Larue de Tournemine & $\begin{array}{l}\text { La complémentarité stratégique entre grandes } \\
\text { entreprises et PME en matière d'innovation } \\
\text { technologique }\end{array}$ & $\begin{array}{l}\text { Revue internationale PME, } \\
1994, \text { vol. } 7, \mathrm{n}^{\circ} 3-4, \text { p. } 173-200 .\end{array}$ \\
\hline $\begin{array}{l}\text { P.-A. Julien, J.-B. Carrière, } \\
\text { L. Raymond et R. Lachance }\end{array}$ & $\begin{array}{l}\text { La gestion du changement technologique } \\
\text { dans la PME manufacturière au Québec: } \\
\text { une analyse de cas multiples }\end{array}$ & $\begin{array}{l}\text { Revue internationale PME, } \\
1994, \text { vol. } 7, \mathrm{n}^{\circ \mathrm{s}} 3-4, \text { p. } 87-120 \text {. }\end{array}$ \\
\hline E.M. Hernandez & L'entrepreneuriat comme processus & $\begin{array}{l}\text { Revue internationale PME, } \\
1995 \text {, vol. } 8, \mathrm{n}^{0} 1, \text { p. } 107-119 .\end{array}$ \\
\hline $\begin{array}{l}\text { B. Saporta et } \\
\text { C. Lapousse-Madrid }\end{array}$ & $\begin{array}{l}\text { Les comportements d'intégration marketing stratégie } \\
\text { et leur influence sur la performance de la petite } \\
\text { entreprise : un cadre conceptuel préliminaire }\end{array}$ & $\begin{array}{l}\text { Revue internationale PME, } \\
1995 \text {, vol. } 8, \mathrm{n}^{\circ} 2 \text {, p. 147-174. }\end{array}$ \\
\hline T. Nobre & $\begin{array}{l}\text { Le processus de structuration dans la petite } \\
\text { entreprise : une étude exploratoire }\end{array}$ & $\begin{array}{l}\text { Revue internationale PME, } \\
1995 \text {, vol. } 8, \mathrm{n}^{0} 2 \text {, p. 204-238. }\end{array}$ \\
\hline G. Paché & Stratégies d'adaptation de la petite entreprise & RFG, 1990 , vol. 78, p. 52-60. \\
\hline J.P. Bréchet & Pour une analyse stratégique adaptée à la PMI & RFG, 1990, vol. 79, p. 19-29. \\
\hline $\begin{array}{l}\text { J.M. Attonaty et } \\
\text { L.G. Soler }\end{array}$ & $\begin{array}{l}\text { Aide à la décision et gestion stratégique: } \\
\text { un modèle pour l'entreprise agricole }\end{array}$ & RFG, 1992, vol. 88, p. 45-54. \\
\hline B. Saporta & Les PME -PMI face au marché unique européen & RFG, 1992, vol. 89, p. 5-16. \\
\hline Y. Lecler & L'avenir du partenariat à la japonaise & RFG, 1992 , vol. 91 , p. $50-63$. \\
\hline J.L. Darréon et S. Faiçal & $\begin{array}{l}\text { Les enjeux des partenariats stratégiques } \\
\text { entre grandes entreprises et PME }\end{array}$ & RFG, 1993, vol. 95, p. 104-115. \\
\hline M. Marchesnay & PME, stratégie et recherche & RFG, 1993, vol. 95, p. 70-76. \\
\hline J.C. Mathé et A. Rivet & Décisions stratégiques et structure de la propriété & RFG, 1993, vol. 95, p. 77-85. \\
\hline J.-G. Degos & $\begin{array}{l}\text { L'image comptable des PME: une grille de lecture } \\
\text { des comportements }\end{array}$ & RFG, 1993, vol. 95, p. 86-95. \\
\hline C. Carrier & $\begin{array}{l}\text { Stratégies intrapreneuriales dans les petites } \\
\text { entreprises }\end{array}$ & RFG, 1993, vol. 95, p. 96-103. \\
\hline A. Fayolle & La trajectoire de l'ingénieur entrepreneur & RFG, 1994, vol. 101, p. 113-125. \\
\hline B. Saporta & La création d'entreprise : enjeux et perspectives & RFG, 1994, vol. 101, p. 74-86. \\
\hline C. Bruyat & $\begin{array}{l}\text { Contributions épistémologiques au domaine } \\
\text { de l'entrepreneuriat }\end{array}$ & RFG, 1994, vol. 101, p. 87-99. \\
\hline E. Vatteville & Le risque successoral & RFG, 1994, vol. 98, p. 18-27. \\
\hline J.P. Bréchet & Du projet d'entreprendre au projet d'entreprise & RFG, 1994, vol. 99, p. 5-14. \\
\hline A. Marchais-Roubelat & Modélisation et complexité : de la décision à l'action & RFG, 1995, vol. 102, p. 102-108. \\
\hline J.C. Ettinger & $\begin{array}{l}\text { Pratiques d'évaluation et de sélection de projets } \\
\text { d'activités nouvelles en Europe }\end{array}$ & RFG, 1995, vol. 102, p. 45-57. \\
\hline Y. Levant & $\begin{array}{l}\text { Peut-on faire de la prévention avec les entreprises } \\
\text { en difficultés? }\end{array}$ & RFG, 1995 , vol. 104, p. 14-23. \\
\hline J.L. Arrègle & $\begin{array}{l}\text { Le savoir et l'approche resource based: } \\
\text { une ressource et une compétence. }\end{array}$ & RFG, 1995 , vol. 105 , p. 84-94. \\
\hline
\end{tabular}




\section{Annexe II}

\section{Grille d'analyse des articles}

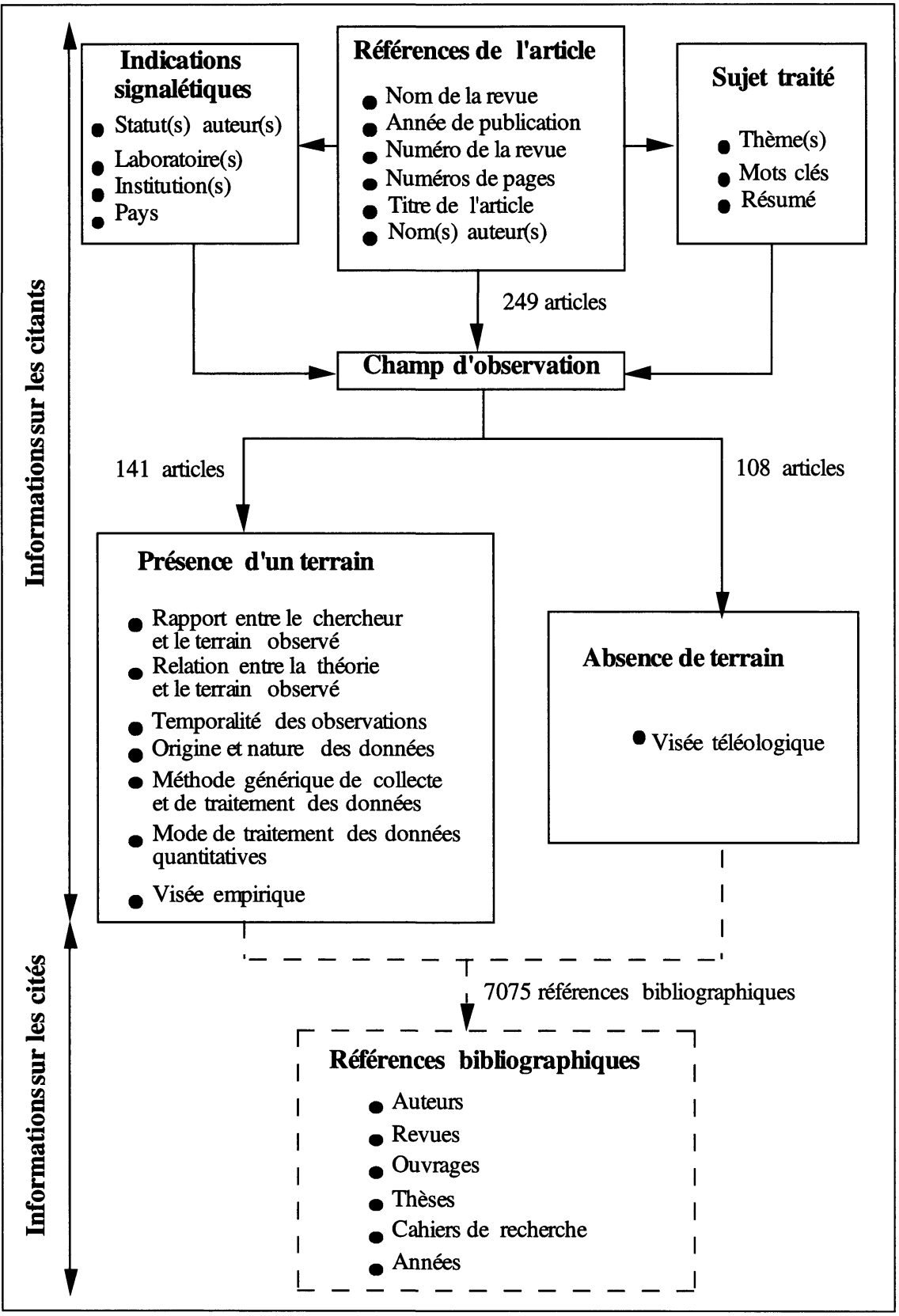




\section{Annexe III}

\section{Méthode des cocitations et construction de réseaux}

Les cocitations d'auteurs sont identifiées et analysées grâce au logiciel Leximappe. L'algorithme fonctionne comme suit (Courtial, de Looze et Penan, 1993):

- Classement des paires d'auteurs par ordre d'association décroissante. Cet ordre est obtenu par le calcul de l'indice de proximité défini comme le produit au carré des probabilités d'avoir un auteur quand on a l'autre, divisé par la fréquence de chacun des auteurs dans le fichier. Avec deux auteurs $i$ et $j$, l'indice de proximité $P i j$ est donc:

$\mathrm{P} i j=(c i j) \_/(c i . c j)$

cij: cooccurrence de $i$ et $j ; c i$ : occurrence de $i ; c j$ : occurrence de $j$.

- Repérage des paires d'auteurs plus fortement associés entre eux qu'avec d'autres. À partir de la première association de la liste, on sélectionne les appariements par ordre décroissant d'intensité. Les autres paires contenant les auteurs retenus dans un agrégat sont alors ignorées pour la construction des autres agrégats. La procédure est renouvelée jusqu'à épuisement de la liste ;

- L'information sur les liens entre les auteurs appartenant à différents agrégats est conservée. Le calcul de la densité (index of internal cohesion) mesure l'intensité des liens entre les auteurs d'un même agrégat. La centralité mesure la valeur moyenne des liens externes d'un agrégat, c'est-à-dire des liens entre les auteurs qui le constituent et les auteurs présents dans d'autres agrégats.

Deux structurations des réseaux ont été obtenues en faisant varier le minima de cocitations. En définissant les réseaux avec un minimum de cinq cocitations, le nombre de réseaux obtenu est réduit (Boissin, Castagnos, Guieu, 1999); avec un minimum de trois cocitations, la représentation est plus extensive. Les ramifications de réseaux sont fines, et pour peu que l' analyste appartienne à la discipline, il donne du sens aux relations et les résultats sont riches. Avec ce minimum de trois cocitations, seule structuration présentée ici, 41 agrégats ont été trouvés par l'algorithme de classification dont 7 sont apparus non significatifs ou fermés (peu de liens externes) et ont donc été éliminés. 


\section{Bibliographie}

BÉCHARD, J.P. (1996), « Comprendre le champ de l'entrepreneurship », Cahier de recherche no 96-01-01, Montréal, HEC.

Boissin J.-P., J.-C. Castagnos et G. Guieu (1997), «Communautés scientifiques et recherche en stratégie », dans Les fondements de la gestion stratégiques, VI Conférence de l'AIMS, Montréal, vol. 1, p. 145-159.

Boissin, J.-P., J.-C. CASTAGnOS et G. GuiEU (1998), «Les fondements transversaux et spécifiques de la recherche francophone en stratégie», dans J.P. Bréchet et $\mathrm{P}$. Mévellec, Valeur, marché et organisation, Nantes, Presses académiques de l'Ouest, vol. 1, p. 475-494.

Boissin, J.-P., J.-C. CASTAGnos et G. GuIEU (1999), «The bibliographic structure of strategic management - a French view », dans Winning Strategy in a Deconstructing World, 19th Annual International Conference of Strategic Management Society, 3-6 octobre, Berlin, 21 p.

Boissin, J.-P., J.-C. CASTAGNOS, G. GuIEU et M.A. DE LOOZE (1999), «La structuration de la recherche francophone en stratégie : une analyse bibliographique », Finance Contrôle - Stratégie, vol. 2, no 3, septembre, p. 63-85.

BOURDIEU, P. (1976), «Le champ scientifique », Actes de la recherche en sciences sociales, vol. $12, n^{\text {os }} 2 / 3$, p. 88-104.

Callon, M., J.P. Courtial et H. Penan (1993), La scientométrie, Paris, Presses universitaires de France, collection «Que sais-je ?».

CASTAGnos, J.-C., J.-P. Boissin et G. GuIEU (1997), « Revues francophones et recherche en stratégie », Économies et sociétés, tome XXXI, n ${ }^{\text {os }} 7-8$, série Sciences de gestion $\mathrm{n}^{\circ} 23$, p. 37-63.

CossetTe, P. (1997), «Les travaux publiés dans Revue internationale PME depuis sa fondation. Caractéristiques et tendances », Revue internationale PME, vol. $10, \mathrm{n}^{\circ} 2$, p. 128.

Courtial, J.P., M.A. DE LOOZE et H. PENAN (1993), « Méthodologie de veille stratégique : application au domaine de la fixation de l'azote», Économie et sociologie rurales, méthodes et instruments, $\mathrm{n}^{\circ} 4$, INRA.

DÉRY, R. (1992), «Enjeux et controverses épistémologiques dans le champ des sciences de l'administration ", Revue canadienne des sciences administratives, vol. $2, \mathrm{n}^{\circ} 1$, p. 1-12.

DÉRY, R. (1996), La structuration socio-historique du champ anglo-saxon de la stratégie », Actes de la journée « Recherche en gestion », Paris, FNEGE, p. 202-251.

DÉRY, R. (1997), « Topographie épistémologique du champ de recherche en management stratégique », Management international, vol. 2, $\mathrm{n}^{\circ}$ 1, p. 11-18.

DÉRY, R. et J.M. TOULOUSE (1996), « Social structuration of the field of entrepreneurship : a case study », Revue canadienne des sciences administratives, vol. 13, no 4, p. 285-305.

FILION, L.J. (1997), «Le champ de l'entrepreneuriat : historique, évolution, tendances », Revue internationale PME, vol. 10, $\mathrm{n}^{\circ}$ 2, p. 129-172. 
JАСОВ, F. (1970), La logique du vivant : une histoire de l'hérédité, Paris, Gallimard.

LATOUR, B. (1989), La science en action, Paris, La Découverte.

MARCheSNAY, M. (1998), "Confiances et logiques entrepreneuriales », Économies et sociétés, série Sciences de gestion, $\mathrm{n}^{\circ} 25$, p. 99-126.

SCHUMPETER, J.A. (1928), «Der Unternehmer», dans Elster Ludwig et al. (dir.), Handwörterbuch der Staatswissenschaften, $4^{\mathrm{e}}$ édition, Berlin, Jena, p. 483.

TORRÈs, O. (1998), «Vingt-cinq ans de recherche en PME : une discipline entre courants et contre-courants », dans $\mathrm{O}$. Torrès (coord.), PME : de nouvelles approches, Paris, Economica, Série Recherche en gestion, chapitre 1, p. 17-53. 